\title{
Chern-Simons and WZW Anomaly Cancelations Across Dimensions
}

\author{
Christopher T. Hill \\ Fermi National Accelerator Laboratory \\ P.O. Box 500, Batavia, Illinois 60510, USA \\ Cosmas K. Zachos \\ High Energy Physics Division, Argonne National Laboratory \\ Argonne, IL 60439-4815, USA
}

(Dated: February 12, 2008)

\begin{abstract}
The WZW functional in $D=4$ can be derived directly from the Chern-Simons functional of a compactified $D=5$ gauge theory and the boundary fermions it supplants. A simple pedagogical model based on $U(1)$ gauge groups illustrates how this works. A bulk-boundary system with the fermions eliminated manifestly evinces anomaly cancelations between CS and WZW terms.
\end{abstract}

PACS numbers: 11.15.-q,12.15.-y,12.38.Qk,12.39.Fe, 13.15.+g,13.40.-f,14.70.Hp,14.80.Mz,95.85.Ry,97.60.Jd

\section{INTRODUCTION}

In this paper we illustrate in a simple scheme how the full Wess-Zumino-Witten (WZW) functional [1, 2] of a gauged chiral lagrangian in $D=4$ arises out of a pure gauge theory of quark flavor in compactified $D=5$. This model, based upon a $U(1)_{L} \times U(1)_{R}$ flavor symmetry, discussed in [3], mimics the chiral structure of QCD, and was used to clarify how the counterterm structure of the WZW functional arises in a parity-asymmetric gauging, such as in the Standard Model.

Here, however, we use it to illustrate how the generic features of the holographic origin of the chiral WZW term arise, where the $D=4$ mesons emerge out of the Wilson line over the bulk gauge field, $A_{5}$. The general gauged WZW functional structure for $U(N) \times U(N)$ has been studied previously using deconstruction [4], while the general gauged Kaymakcalan, Rajeev, and Schechter, (KRS) action [5] has been derived from continuum compactification of a pure $S U(N)$ Yang-Mills theory in detail in [6]. The present work is intended, in part, to clarify the approach and results of $[6]$.

We construct a manifestly $U(1)$ gauge-invariant theory in $D=5$. The gauge fields propagate in the bulk, with chiral quarks attached to chiral boundaries (branes), with $L(R)$ located at $x^{5}=0\left(x^{5}=R\right)$, respectively. The quarks are chirally delocalized in $D=5$ [7], and their chiral anomalies [8] are nonzero on their respective boundaries, but would otherwise cancel if the boundaries were merged.

The boundary conditions on the $D=5$ gauge fields are subject to a minimal set of constraints: (I) there exists a massless physical $A_{5}$ zero mode, (or, more properly, a nontrivial Wilson line spanning the bulk) which can be identified with chiral mesons; and, (II) there exists a tower of KK modes of the gauge fields, which is sufficiently rich, such that independently valued combinations of these fields exist on the boundary branes.

Much of what we cover in our pedagogical model is expected to apply to any theory of new physics in extra dimensions which satisfies (I-II) with chiral delocaliza- tion, including $\operatorname{AdS} D=5$ models. Irrespective of the specific $D=5$ geometry, our low-energy effective theory results are holographic, i.e., they are determined at the boundary, as the integrands in the bulk involving the lower KK-modes are mostly exact expressions. Since the theory we consider is arranged to be anomaly free, the resulting effective action contains both the holographic WZW, and a dual effective interaction in the bulk. This latter bulk interaction takes the form of a Chern-Simons (CS) functional [9, 10] in the low energy effective theory variables [6, 7], and cancels the anomalies on the boundaries.

With the chiral quarks attached to the boundaries, $\psi_{L}$ at $x^{5}=0$, and $\psi_{R}$ at $x^{5}=R$, respectively, a "constituent quark mass term" is introduced of the form $m \bar{\psi}_{L} W \psi_{R}+h . c$. . Here, $W$ is the Wilson line that spans the gap between the boundary branes, and represents the dynamical chiral condensate of the theory. The Wilson line is identified with a chiral field of mesons:

$$
W\left(x^{\mu}\right)=\exp \left(i \int_{0}^{R} d x^{5} A_{5}\left(x^{\mu}, x^{5}\right)\right) \equiv \exp \left(\frac{i a\left(x^{\mu}\right)}{f}\right) .
$$

This is the rationale for requiring an $A_{5}$ zero mode, since we need that the pseudoscalar chiral meson $a$ be physical, and not be eaten by a KK-mode. The chiral symmetry breaking scale is specified by $f$, the "pion decay constant"; in any imitation of QCD chiral dynamics by an extra dimension, chiral symmetry breaking is generically related to the compactification scale, i.e., $f \sim 1 / R$.

The quark anomalies [8] on the boundary branes must be canceled by the anomalies that arise on the boundaries from a bulk-filling Chern-Simons functional. The anomaly cancellation condition determines the coefficient of the Chern-Simons functional [10]. We integrate out the quarks, taking the limit of large $m$. In a special gauge, an effective "Fujikawa" action arises out of the quark Dirac determinant 11], which only involves the gauge fields on the boundary, and amounts to minus the Bardeen counterterm [3, [5, 8].

When the Chern-Simons term and the boundary term are added together, they yield a total effective action, 
$S^{*}$, all of whose anomalies cancel. Through a suitable reverse gauge transformation, we finally arrive at the purely bosonic anomaly-free action, consisting of a surface term and bulk term,

$S^{*}=\Gamma_{W Z W}\left(a\left(x^{\mu}\right), A_{L}\left(x^{\mu}\right), A_{R}\left(x^{\mu}\right)\right)+S_{C S}\left(A_{A}\left(x^{\mu}, x^{5}\right)\right)$.

$\Gamma_{W Z W}\left(a, A_{L}, A_{R}\right)$ is the full (gauged) WZW functional, on the boundary. $S_{C S}$ is the original bulk Chern-Simons functional, whose anomalies cancel those of $\Gamma_{W Z W}$.

For a purely $D=4$ theory such as QCD, we would have to discard the bulk term $S_{C S}$ and leave behind the WZW term $\Gamma_{W Z W}$, whose anomalies would then need be canceled by, e.g., suitable leptons, instead. In a generic AdS-CFT correspondence setting, we would have both the $\Gamma_{W Z W}$ and the $S_{C S}$ effective interactions to generate anomalies. $S_{C S}$, living in the bulk, also contains the KK modes of the theory, and leads to a richer system of new degrees of freedom. These were previously studied in detail for QED in refs [6, 7], whose basic procedure is effectively exemplified here.

We stress that this approach is significantly different from the standard dimensional descent approach, pioneered by Witten [2]. In that approach, a chiral theory of mesons in $D=5$ is considered from the start, and a $D=5$ chirally invariant closed but not exact pionic interaction, $\operatorname{Tr}(d \pi d \pi d \pi d \pi d \pi)+\ldots$ with a quantized coefficient is introduced. Dimensional descent to $D=4$ yields the boundary term $\operatorname{Tr}(\pi d \pi d \pi d \pi d \pi)+\ldots$, which is subsequently gauged, a posteriori.

By contrast, our present procedure begins with a pure gauge theory and is a priori gauge invariant. The mesons emerge into being in the descent by compactification out of bulk gauge-field Wilson lines, [4]. We rely on the CS interaction to produce the full WZW term, with its meson and gauge field components suitably linked - and possibly connected by further, still unappreciated, symmetries. In this sense, our approach would fit within the broad dimensional descent framework of ref [10], conforming to its abstract mechanisms, and has analogies with the bulk - string anomaly-cancellation mechanisms of [12] and [13], which rely on chiral zero modes of fermions on axionic-defect submanifolds. Our straightforward model is particularly revealing, as now both mesons and gauge fields are part of the same construct.

We start by reviewing the pedagogical toy model and its canceling anomaly structure in $D=4$ in Section II. We proceed to derive the full WZW term exploiting interplay with the $D=5$ bulk in Section III, and discuss implications of the picture introduced in Section IV.

\section{SCHEMATIC STANDARD MODEL WITH CHIRAL PSEUDOSCALAR INTERACTIONS}

We imitate the standard model with a simple $U(1) \times$ $U(1)$ gauge theory, discussed previously in ref [3]. Consider a single color $\left(N_{c}=1\right)$ and flavor of "quark," $q$, and a single "lepton," $\ell$. Introduce $U(1)_{L}$ and $U(1)_{R}$ fundamental gauge fields $A_{L}$ and $A_{R}$ into the quark action:

$$
S_{q}=\int d^{4} x \bar{q}_{L}\left(i \not \partial-A_{L}\right) q_{L}+\bar{q}_{R}\left(i \not \supset-\not A_{R}\right) q_{R} .
$$

Likewise, we gauge the "lepton" sector:

$$
S_{\ell}=\int d^{4} x \bar{\ell}_{L}\left(i \not \partial+\not A_{L}\right) \ell_{L}+\bar{\ell}_{R}\left(i \not \partial+A_{R}\right) \ell_{R} .
$$

Note the sign changes of the couplings. Taken together, the gauge anomalies cancel between the quark and lepton sectors in the $B-L$ currents,

$$
\begin{aligned}
& \partial_{\mu}\left(\bar{q} \gamma^{\mu} q_{L}-\bar{\ell} \gamma^{\mu} \ell_{L}\right)=\partial^{\mu} J_{L \mu}^{B-L}=0, \\
& \partial_{\mu}\left(\bar{q} \gamma^{\mu} q_{R}-\bar{\ell} \gamma^{\mu} \ell_{R}\right)=\partial^{\mu} J_{R \mu}^{B-L}=0 .
\end{aligned}
$$

Anomalies remain in the ungauged $B+L$ currents, imitating the structure of the standard model. (The leptons are here only to cancel anomalies, and this role will be assumed, in the next section, by other, CS, bulk gauge interactions.)

One may see the analogy to hadronic physics and the chiral lagrangian of QCD. This may be done by decoupling the quarks made heavy by spontaneously breaking the $U(1)_{L} \times U(1)_{R}$ symmetry to $U(1)$. Doing so introduces an effective constituent quark mass term containing a pseudoscalar chiral Nambu-Goldstone boson,

$$
m \bar{q}_{L} q_{R} e^{i a / f}+\text { h.c. }
$$

$a\left(x^{\mu}\right) / f$ is the analog in our system of $\pi^{0} / f_{\pi}$ in QCD. Under the $U(1)_{L} \times U(1)_{R}$ gauge transformations, we have for the quark sector:

$$
\begin{array}{lll}
q_{L} \rightarrow e^{i \epsilon_{L}} q_{L} & \delta A_{L}=-d \epsilon_{L} & \delta a=f \epsilon_{L} \\
q_{R} \rightarrow e^{i \epsilon_{R}} q_{R} & \delta A_{R}=-d \epsilon_{R} & \delta a=-f \epsilon_{R} .
\end{array}
$$

(The leptons likewise transform as $\ell_{L} \rightarrow e^{-i \epsilon_{L}} \ell_{L}$ and $\left.\ell_{R} \rightarrow e^{-i \epsilon_{R}} \ell_{R}\right)$. These gauge transformations are anomalous, shifting the original quark sector action by the consistent anomalies [14],

$$
\delta S_{q}=\frac{1}{24 \pi^{2}} \int d^{4} x\left(-\epsilon_{L} d A_{L} d A_{L}+\epsilon_{R} d A_{R} d A_{R}\right) .
$$

They are, of course, canceled by $\delta S_{\ell}$. We'll utilize compact differential form notation, e.g.,

$$
\int A B d C=\int d^{4} x \epsilon^{\mu \nu \rho \sigma} A_{\mu} B_{\nu} \partial_{\rho} C_{\sigma} .
$$

In principle, the large $m$ limit of integrating the quarks out (which, for anomalies, in a sense, parallels confinement) results in an effective action [15], which is a functional of the $U=e^{i a\left(x^{\mu}\right) / f}$ and the gauge fields, $A_{L}$ and $A_{R}, \Gamma_{W Z W}\left(U, A_{L}, A_{R}\right)$. This functional generates the same anomalies as in eq.(8), and it is the WZW functional of our theory, which codifies the anomaly effects of the entire quark sector. 
However, in practice, it is easy to construct the parityinvariant WZW term heuristically, by arranging a set of $D=4$ operators that generate the independent $L$ and $R$ consistent anomalies. One readily obtains

$$
\begin{aligned}
\Gamma_{W Z W} & =-\frac{1}{24 \pi^{2}} \int d^{4} x\left[A_{R} A_{L} d A_{L}-A_{L} A_{R} d A_{R}\right. \\
& \left.+\frac{a}{f}\left(d A_{L} d A_{L}+d A_{R} d A_{R}+d A_{L} d A_{R}\right)\right]
\end{aligned}
$$

Under the gauge transformations of eq.(17), we obtain precisely $\delta \Gamma_{W Z W}=\delta S_{q}$ of eq.(8). That is, $\Gamma_{W Z W}$ is indeed the WZW part of the full action of the low-energy hadronic theory [16]. In the $f \rightarrow \infty$ limit in which the pseudoscalar $a\left(x^{\mu}\right)$ decouples, $\Gamma_{W Z W}\left(\mathbb{1}, A_{L}, A_{R}\right) \equiv \Gamma_{0}$ reduces to the Bardeen counterterm. (The role of the meson is no more visible in $\Gamma_{W Z W}$ for $\epsilon_{L}=\epsilon_{R}$.)

In conclusion, the action $S_{\ell}+\Gamma_{W Z W}$ is gauge invariant.

\section{HOLOGRAPHIC ORIGINS OF THE WZW TERM}

\section{A. The Setup}

Here, we derive eq. (10) by embedding the theory into a $D=5 U(1)$ gauge theory. Denote $x^{5} \equiv y$. For simplicity, the bulk dimensionful constants have been absorbed into the definition of $y$ and $A_{A}$. In this theory, we embed chirally delocalized fermions on boundary $D=4$-branes $I$ and $I I$. On brane I, at $y=0$, we have:

$$
S_{I}=\int_{I} d^{4} x \bar{q}_{L}\left(i \not \partial-\not A_{L}\right) q_{L},
$$

and, on brane II at $y=R$,

$$
S_{I I}=\int_{I I} d^{4} x \bar{q}_{R}\left(i \not \partial-\not A_{R}\right) q_{R} .
$$

The $U(1)$ gauge field $A_{A}\left(x_{\mu}, y\right)$ propagates in the $D=5$ bulk, and

$$
A_{\mu L}=A_{\mu}\left(x_{\mu}, 0\right), \quad A_{\mu R}=A_{\mu}\left(x_{\mu}, R\right) .
$$

To cancel the anomalies in this theory, instead of $S_{\ell}$ above, we introduce into the bulk the $D=5$ ChernSimons functional [10],

$$
S_{C S}=c \int d^{4} x \int_{0}^{R} d y \epsilon_{A B C D E} A^{A} \partial^{B} A^{C} \partial^{D} A^{E},
$$

where $c$ is quantized by general arguments, [9, 10],

$$
c=\frac{1}{24 \pi^{2}} \text {. }
$$

Further consider the abelian Wilson line,

$$
W=\exp \left(i \int_{0}^{R} d y A_{5}\left(x_{\mu}, y\right)\right)
$$

This permits construction of a bilocally gauge-invariant fermion mass term,

$$
S_{m}=-\int d^{4} x m \bar{q}_{L}\left(x_{\mu}\right) W q_{R}\left(x_{\mu}\right)+\text { h.c. }
$$

We identify the line integral with the boundary meson,

$$
\int_{0}^{R} d y A_{5}\left(x_{\mu}, y\right) \equiv \phi\left(x_{\mu}\right) \equiv a\left(x_{\mu}\right) / f
$$

even though, for formal convenience, below, we will utilize a bulk generalization,

$$
\int_{0}^{y} d z A_{5}\left(x_{\mu}, z\right) \equiv \Phi\left(x_{\mu}, y\right),
$$

such that $\Phi\left(x_{\mu}, 0\right)=0$, and $\Phi\left(x_{\mu}, R\right)=\phi\left(x_{\mu}\right)$ on the chiral boundaries. In the localized limit, $R \rightarrow 0, f \rightarrow \infty$, and the chiral boson drops out of the theory.

\section{B. Anomalies}

The actions $S_{I}$ and $S_{I I}$ have anomalies on their respective branes. Under the chiral boundary gauge transformations,

$$
\begin{aligned}
q_{L} & \rightarrow e^{i \epsilon_{L}} q_{L} \\
q_{R} \rightarrow e^{i \epsilon_{R}} q_{R} & A_{L} \rightarrow A_{L}-d \epsilon_{L} \\
A_{R} & \rightarrow A_{R}-d \epsilon_{R},
\end{aligned}
$$

we have:

$$
\begin{aligned}
& \delta S_{I}=-\frac{1}{24 \pi^{2}} \int d^{4} x \epsilon_{L} d A_{L} d A_{L} \\
& \delta S_{I I}=\frac{1}{24 \pi^{2}} \int d^{4} x \epsilon_{R} d A_{R} d A_{R}
\end{aligned}
$$

The mass term, as reviewed below, does not affect the full quark answer,

$$
\delta S_{q}=\delta S_{I}+\delta S_{I I}
$$

In the bulk, the full gauge transformation is

$$
\begin{aligned}
A_{A} \rightarrow & A_{A}-\partial_{A} \epsilon\left(x_{\mu}, y\right) \\
& \epsilon\left(x_{\mu}, 0\right)=\epsilon_{L}, \quad \epsilon\left(x_{\mu}, R\right)=\epsilon_{R} .
\end{aligned}
$$

It is useful to decompose the Chern-Simons term into $A_{5}$ and $\partial_{5}=\partial_{y}$ components, expressed as 4 -forms,

$$
S_{C S}=c \int d^{4} x d y\left[3 A_{5} d A d A-2 A\left(\partial_{y} A\right) d A\right] .
$$

Under the gauge transformation of eq.(23), $S_{C S}$ generates the anomalies on the boundaries [9, 10]. (Naturally, there are no anomalies in the bulk, as odd-dimensional fermions lack the chirality to produce such.) In what follows, it is useful to display this result for the form chosen 
in eq.(24),

$$
\begin{aligned}
\delta S_{C S}= & c \int d^{4} x d y\left[3\left(-\partial_{y} \epsilon\right) d A d A+2(d \epsilon)\left(\partial_{y} A\right) d A\right. \\
& \left.+2 A\left(\partial_{y} d \epsilon\right) d A\right] \\
= & -c \int d^{4} x d y \partial_{y}(\epsilon d A d A) \\
= & \frac{1}{24 \pi^{2}} \int d^{4} x\left[\epsilon_{L} d A_{L} d A_{L}-\epsilon_{R} d A_{R} d A_{R}\right] \\
= & -\delta S_{q} .
\end{aligned}
$$

Thus, the full action,

$$
S^{*}=S_{I}+S_{I I}+S_{m}+S_{C S},
$$

amounts to a gauge-invariant theory,

$$
\delta S^{*}=0,
$$

under the general gauge transformation, eq.(23).

Now note that the mesonless, localized $(R=0 \Leftrightarrow f=$ $\infty)$ quark theory with $W=1$ is a $D=4$ action,

$$
\begin{gathered}
S_{0}=\int d^{4} x\left[\bar{q}_{L}\left(i \not \partial-A_{L}\right) q_{L}+\bar{q}_{R}\left(i \not \partial-A_{R}\right) q_{R}\right. \\
\left.-m\left(\bar{q}_{L} q_{R}+\text { h.c. }\right)\right] .
\end{gathered}
$$

If we integrate out the fermions and expand in $1 / m$ the logarithm of the resulting fermion determinant, $\operatorname{Tr} \log (\mathbb{1}-i \not D / m)$, to leading order [11], we obtain an operator functional of $A_{L}$ and $A_{R}$ of the form,

$$
\begin{aligned}
\Gamma_{0} & =-\frac{1}{6 \pi^{2}} \int d^{4} x A V d V \\
& =\frac{1}{24 \pi^{2}} \int d^{4} x\left[A_{L} A_{R} d A_{L}+A_{L} A_{R} d A_{R}\right],
\end{aligned}
$$

where $A_{R}=V+A$ and $A_{L}=V-A$.

Thus, the sole surviving influence of the fermions removed from play is the effective term, eq.(29), which amounts to minus the Bardeen counterterm. If we added the Bardeen counterterm to $S_{0}$, we would cancel $\Gamma_{0}$ to thus obtain a vanishing result. This reflects the anomaly decoupling. The covariant axial and vector currents are defined by (I) adding the Bardeen counterterm to the action and (II) then varying the action with respect to either $V$ or $A$. The matrix elements of these currents can be seen to vanish in the large $m$ limit. Therefore, by adding the Bardeen counterterm to the action, and then integrating out the massive fermions, the resulting action vanishes, and the current matrix elements are not generated at all.

To be more precise, consider the covariant axial anomaly equation:

$$
\partial_{\mu} j^{\mu 5}+2 i m \bar{q} \gamma^{5} q=\frac{1}{16 \pi^{2}}\left(d V d V+\frac{1}{3} d A d A\right) .
$$

The matrix elements of this equation are saturated on the lhs by the $2 i m \bar{q} \gamma^{5} q$ term, which survives in the large $m$ limit; while the matrix elements of $j^{\mu 5}$ are vanishing like $1 / \mathrm{m}^{2}$. These latter matrix elements, which would be obtained by variation with respect to $A$, are simply not generated when $\Gamma_{0}$ is canceled by the counterterm.

\section{A Gauge Transformation}

Our strategy is to exploit the gauge invariance of $S^{*}$, and to go to an axial gauge, in which $A_{5}=0$ and $W=1$, in which we can integrate the fermions out as above, and to then revert to the original gauge.

To this end, we introduce a gauge transformation in the bulk,

$$
\begin{gathered}
U(x, y)=\exp \left(-i \int_{0}^{y} d z A_{5}\left(x_{\mu}, z\right)\right), \\
B_{A}(x, y) \equiv A_{A}(x, y)-\partial_{A} \Phi(x, y) \\
\partial_{A} \Phi=i U(x, y)^{\dagger} \partial_{A} U(x, y),
\end{gathered}
$$

and, on the boundaries, $\Phi(x, 0)=0, \Phi(x, R)=\phi(x)$ and

$$
\psi_{L} \rightarrow U(x, 0) \psi_{L}, \quad \psi_{R} \rightarrow U(x, R) \psi_{R} .
$$

This transformation implies $B_{5}=0$, an axial gauge, thus fixing $W=1$ in $S_{m}$, while $S_{C S}$ reduces to

$$
S_{C S}^{0}=-2 c \int d^{4} x d y B \partial_{y} B d B .
$$

Thus, we have

$$
B_{\mu}(x, 0)=A_{L \mu}(x), \quad B_{R \mu}(x)=A_{R \mu}(x)-\partial_{\mu} \phi(x) .
$$

The gauge transformation chosen is parity-asymmetric, with $\phi$ appearing in $A_{R}$, but absent in $A_{L}$. (We could have chosen a more parity-symmetric form, e.g., defining $U=1$ at $y=1 / 2$, but our present choice has the advantage of providing an internal consistency check on the interplay between the fermion loop $\left(\Gamma_{0}\right)$ and the ChernSimons term in the final result.) The overall result will be parity symmetric.

On the $B_{\mu}=A_{\mu}-\partial_{\mu} \Phi(x, y)$ bulk components, this gauge transformation has provided longitudinal components for all of the massive $K K$-modes (unitary gauge), and allows them to be treated as Stueckelberg fields, $B_{\mu}^{n}=A_{\mu}^{n}-\partial_{\mu} \phi^{n}$. However, significantly, the $y$ independent (zero) mode, which is the Wilson line integral over $A_{5}$, provides a residual uneaten $\phi$-field zero mode.

\section{Derivation of the WZW Term}

With the effective removal of the phase in the Wilson line, $W$, we now have the effective quark action,

$$
\begin{aligned}
S_{0}= & \int d^{4} x \bar{q}_{L}\left(i \not \partial-\not{ }_{L}\right) q_{L}+\bar{q}_{R}\left(i \not \partial-B_{R}\right) q_{R} . \\
& -m\left(\bar{q}_{R} q_{L}+\text { h.c. }\right) .
\end{aligned}
$$


Integrating out the quarks in the large $m$ limit, as reviewed above, we obtain the effective action from eq.(29),

$$
\Gamma_{0}=\frac{1}{24 \pi^{2}} \int d^{4} x\left[B_{L} B_{R} d B_{L}+B_{L} B_{R} d B_{R}\right] .
$$

This is, of course, not separately gauge invariant. The gauge invariant action is

$$
S^{*}=\Gamma_{0}+S_{C S}^{0}
$$

Now, performing the inverse gauge transformation, (35), from $B$ to $A$, yields, on the one hand,

$$
\begin{aligned}
\Gamma_{0}= & \frac{1}{24 \pi^{2}} \int d^{4} x\left[A_{L} A_{R} d A_{L}+A_{L} A_{R} d A_{R}\right. \\
& \left.+d \phi\left(A_{L} d A_{L}+A_{L} d A_{R}\right)\right] .
\end{aligned}
$$

Note the appearance of $d \phi$ with $A_{R}$, but not $A_{L}$, a consequence of our parity-asymmetric gauge transformation choice, so the expression is not parity symmetric.

On the other hand, the gauge-variant bulk term (34) now also transforms under the same inverse transformation to

$$
S_{C S}^{0}=-2 c \int d^{4} x d y(A-d \Phi) \partial_{y}(A-d \Phi) d A .
$$

This expression may be recast through $\partial_{y} \Phi=A_{5}$ as

$$
S_{C S}^{0}=-c \int d^{4} x d y\left[2 A\left(\partial_{y} A-d A_{5}\right) d A+\Phi \partial_{y}(d A d A)\right] .
$$

Through an integration by parts, we recover the bulk term (24) and an additional boundary term,

$$
\begin{aligned}
S_{C S}^{0} & =c \int d^{4} x d y\left(3 A_{5} d A d A-2 A\left(\partial_{y} A\right) d A\right) \\
& -c \int d^{4} x d y \partial_{y}(\Phi d A d A) \\
& =S_{C S}+\delta S^{0} .
\end{aligned}
$$

The boundary piece is also parity asymmetric,

$$
\delta S^{0}=-\frac{1}{24 \pi^{2}} \int d^{4} x\left[\phi d A_{R} d A_{R}\right]
$$

and, when added to above fermion determinant boundary contribution, (39), yields the full, parity-symmetric, gauge-noninvariant WZW action, (10), on the boundary branes,

$$
\begin{aligned}
\Gamma_{W Z W}= & -\frac{1}{24 \pi^{2}} \int d^{4} x\left[A_{R} A_{L} d A_{L}-A_{L} A_{R} d A_{R}\right. \\
& \left.+\phi\left(d A_{L} d A_{L}+d A_{R} d A_{R}+d A_{L} d A_{R}\right)\right] .(44)
\end{aligned}
$$

To restore proper dimensions, take $\phi=a / f$.

So it is the interplay of the fermion determinant with the holographic gauge-invariance violation of the bulk CS term which gives rise to the full gauge-invariant effective action. This action then,

$$
S^{*}=\Gamma_{W Z W}+S_{C S}
$$

amounts to the boundary WZW term and the bulk CS term, which cancel each other's anomalies [17].

Consequently, either the boundary or (minus) the bulk terms are equivalent bosonic representations of the very same boundary fermion anomalies, and may thus be effectively regarded as holographic duals to each other [4].

\section{IMPLICATIONS}

The significance of dimensionally-descending bosonic functionals in anomaly physics has been long appreciated [2, 10]. Moreover, even though superficially very different, the CS functionals in odd spacetime dimensions and the WZW functionals in even ones are, in fact, linked in a unique structure [4].

Nevertheless, within the context of actual brane models, the ready holographic cancellation of anomalies by the respective bulk CS and boundary WZW functionals identified here, and, indeed, the effective dual representation of the very same anomalies, is particularly transparent, and perhaps useful to model-building. Loosely, one may think of the WZW term as the holographic AdS/CFT image of the CS functional.

To be sure, the present simple model deals with anomaly inflows specified by fermions strictly confined to boundary branes. Given the holographic projection identified here, however, one is justified to ask whether fermions incompletely localized on boundary branes [12, 13], developing chiral zero modes on the boundary, would also be amenable to analogous treatment, if integrated out to a bosonic effective action. In odd spacetime dimensions, there are no chiral fermions leading to anomalies, but they may develop chiral zero modes on evendimensional submanifolds. In such a theory, would the higher KK modes, which did not couple to the WZW functional here (but which are present, inertly, in the CS functional in our present treatment), insinuate themselves into a generalization of the full (gauged) WZW term derived? The issue is under current investigation.

\section{Acknowledgments}

We thank Jeff Harvey and Richard Hill for helpful discussions. Research supported by the U.S. Department of Energy grant DE-AC02-76CHO3000, and by the U.S. Department of Energy, Division of High Energy Physics, Contract DE-AC02-06CH11357 as well as the University of Chicago - Argonne Joint Theory Institute. 
[1] J. Wess and B. Zumino, "Consequences of anomalous Ward identities," Phys. Lett. B 37, 95 (1971).

[2] E. Witten, "Global Aspects Of Current Algebra," Nucl. Phys. B 223, 422 (1983).

[3] J. A. Harvey, C. T. Hill and R. J. Hill, "Standard Model Gauging of the WZW Term: Anomalies, Global Currents and pseudo-Chern-Simons Interactions," arXiv:0712.1230 [hep-th].

J. A. Harvey, C. T. Hill and R. J. Hill, "Anomaly mediated neutrino-photon interactions at finite baryon density," arXiv:0708.1281 [hep-ph], Phys. Rev. Lett., in press.

[4] C. T. Hill and C. K. Zachos, "Dimensional deconstruction and Wess-Zumino-Witten terms," Phys. Rev. D 71, 046002 (2005).

[5] O. Kaymakcalan, S. Rajeev and J. Schechter, "Nonabelian Anomaly And Vector Meson Decays," Phys. Rev. D 30, 594 (1984).

J. L. Mañes, "Differential Geometric Construction Of The Gauged Wess-Zumino Action," Nucl. Phys. B 250, 369 (1985);

N. K. Pak and P. Rossi, "Gauged Goldstone Boson Effective Action From Direct Integration Of Bardeen Anomaly," Nucl. Phys. B 250, 279 (1985).

[6] C. T. Hill, "Exact equivalence of the D $=4$ gauged WessZumino-Witten term and the D $=5$ Yang-Mills ChernSimons term," Phys. Rev. D 73, 126009 (2006);

[7] C. T. Hill, "Anomalies, Chern-Simons terms and chiral delocalization in extra dimensions," Phys. Rev. D 73, 085001 (2006).

[8] J. Steinberger, "On the use of subtraction fields and the lifetimes of some types of meson decay," Phys. Rev. 76, 1180 (1949).

J. S. Bell and R. Jackiw, "A PCAC puzzle: $\pi^{0} \rightarrow \gamma \gamma$ in the sigma model," Nuovo Cim. A 60, 47 (1969).

S. Adler, "Axial vector vertex in spinor electrodynamics," Phys. Rev. 177, 2426 (1969). W. A. Bardeen, "Anomalous Ward Identities In Spinor Field Theories," Phys. Rev. 184, 1848 (1969).

[9] S. Deser, R. Jackiw and S. Templeton, "Topologically massive gauge theories," Annals Phys. 140, 372 (1982) [Erratum-ibid. 185, 406.1988 ; S. Deser, R. Jackiw and S. Templeton, "Three-Dimensional Massive Gauge Theo- ries," Phys. Rev. Lett. 48, 975 (1982); Y. S. Wu, "ChernSimons Topological Lagrangians In Odd Dimensions And Their Kaluza-Klein Reduction," Annals Phys. 156, 194 (1984).

[10] B. Zumino, Y. S. Wu and A. Zee, "Chiral Anomalies, Higher Dimensions, And Differential Geometry," Nucl. Phys. B 239, 477 (1984).

[11] K. Fujikawa, "Path Integral For Gauge Theories With Fermions," Phys. Rev. D 21, 2848 (1980) [Erratum-ibid. D 22, 1499 (1980)].

[12] C. Callan and J. A. Harvey, "Anomalies And Fermion Zero Modes On Strings And Domain Walls," Nucl. Phys. B 250, 427 (1985).

[13] D. B. Kaplan, "A Method for simulating chiral fermions on the lattice," Phys. Lett. B 288, 342 (1992) arXiv:hep-lat/9206013 ; M. Stone and F. Gaitan, "Topological Charge and Chiral Anomalies in Fermi Superfluids," Annals Phys. 178, 89 (1987); N. Kopnin, G. Volovik and U. Parts, "Spectral Flow in Vortex Dynamics of 3He-B and Superconductors," Europhys. Lett. 32, 651 (1995) arXiv:cond-mat/9509157; W. Liao, "Anomaly inflow mechanism using Wilson line," Phys. Rev. D 75, 065007 (2007) arXiv:hep-th/0605134; B. Gripaios and S. M. West, "Anomaly Holography," Nucl. Phys. B 789, 362 (2008) arXiv:0704.3981 [hep-ph]].

[14] W. A. Bardeen and B. Zumino, "Consistent And Covariant Anomalies In Gauge And Gravitational Theories," Nucl. Phys. B 244, 421 (1984).

[15] E. D'Hoker and E. Farhi, "Decoupling A Fermion Whose Mass Is Generated By A Yukawa Coupling: The General Case," Nucl. Phys. B 248, 59 (1984).

[16] Note that one could also formally obtain eq. (10) from the full $U(N)_{L} \times U(N)_{R}$ WZW functional specified by Kaymakcalan, Rajeev and Schechter (KRS) [5], by taking its $U(1)_{L} \times U(1)_{R}$ limit. That full functional results out of coupling $\Gamma(U, 0,0)_{W Z W}$ to gauge fields, but for the abelian subgroups in our limit, $\Gamma(U, 0,0)_{W Z W}=0$.

[17] Strictly speaking, in principle, the above straighforward derivation could have been duplicated by brute force application of the procedure of ref [15], i.e., direct integration out of the quarks, although the presence of the pseudoscalar meson would have complicated the evaluation of the functional determinant. 